\title{
Ganho genético decorrente da substituição anual de cultivares de milho
}

\author{
Lindolfo Storck ${ }^{(1)}$, Dílson Antônio Bisognin(1) e Alberto Cargnelutti Filho(2)
}

\begin{abstract}
(1)Universidade Federal de Santa Maria, Dep. de Fitotecnia, Avenida Roraima s/no, Bairro Camobi, CEP 97105-900 Santa Maria, RS. E-mail: lindolfo@smail.ufsm.br, dilsonb@ccr.ufsm.br (2)Fundação Estadual de Pesquisa Agropecuária, Rua Gonçalves Dias, no 570, Bairro Menino Deus, CEP 90130-060 Porto Alegre, RS. E-mail: alberto-cargnelutti@fepagro.rs.gov.br
\end{abstract}

Resumo - Cultivares de milho (Zea mays) são normalmente substituídas alguns anos após a indicação para cultivo. Os objetivos deste trabalho foram desenvolver e avaliar um teste de hipótese para determinar se as substituições anuais de cultivares, nos ensaios de competição de cultivares indicadas de milho, resultam em ganho genético para produtividade de grãos. Os ensaios de competição de cultivares de milho superprecoce, precoce e normal foram conduzidos nos anos agrícolas de 1998/1999, 1999/2000, 2000/2001 e 2001/2002. O delineamento experimental foi em blocos ao acaso, com três repetições. Cada parcela foi formada por duas fileiras de $5 \mathrm{~m}$ de comprimento, com espaçamento de $0,8 \mathrm{~m}$. O ganho genético, quanto à produtividade de grãos, foi estimado pelo método proposto por Vencovsky et al., e a significância verificada pelo teste de hipótese proposto neste trabalho. As substituições anuais de cultivares de milho são dinâmicas e nem sempre resultam em ganho genético para produtividade de grãos.

Termos para indexação: Zea mays, melhoramento, teste de hipótese.

\section{Genetic gain as a result of corn cultivars annual substitution}

\begin{abstract}
Corn (Zea mays) cultivars are usually replaced a few years after release. The objective of this study was to evaluate a proposed hypothesis test to determine if the annually cultivars replacements, in comparative field trials of recommended corn cultivars, result in genetic gain of grain yield. Comparative field trials of super early, early and normal corn cultivars were carried out during 1998/1999, 1999/2000, 2000/2001 and 2001/2002 grown seasons. The experimental design was a complete randomized block with three replications. Each plot had two rows of $5 \mathrm{~m}$ separated by $0.8 \mathrm{~m}$. Grain yield genetic gain was estimated by the Vencovsky et al. method. Genetic gain significance was verified with the proposed hypothesis test. The annual replacement of corn cultivars is a dynamic process that not always result in genetic gain of grain yield.
\end{abstract}

Index terms: Zea mays, breeding, hypothesis test.

\section{Introdução}

As redes de ensaios de competição de cultivares de milho (Zea mays) fazem parte das etapas finais de um programa de melhoramento genético. Nesses ensaios, as cultivares que não apresentam bom desempenho são descartadas e substituídas por outras, supostamente superiores, que possam proporcionar um progresso genético contínuo. Os resultados subsidiam critérios para a indicação de cultivares. Além disso, as informações obtidas são utilizadas pelos técnicos e produtores, para a escolha das cultivares a serem adotadas na produção de milho em cada região. No entanto, uma vez que determinada cultivar é indicada, é mantida no mercado de sementes de forma liberal, já que a decisão de produzir sementes de determinada cultivar é exclusiva das empresas melhoradoras ou detentoras dos direitos de multiplicação. Em conseqüência, existe uma elevada taxa de substituição anual de cultivares indicadas (Storck et al., 2000).

A proporção da área cultivada com determinada cultivar, os ensaios das cultivares lançadas em diferentes épocas e o emprego dos resultados de ensaios regionais de competição de cultivares são procedimentos usados para estimar o ganho genético de uma determinada cultura, em um programa de melhoramento. O uso da proporção da área cultivada com determinada cultivar, como forma de medir a eficiência de um programa de melhoramento, pode não representar apenas o avanço genético, pois a adoção de uma cultivar envolve não apenas o 
potencial genético como também a habilidade da empresa no processo de difusão (Abreu et al., 1994). A quantificação do progresso genético, envolvendo a comparação de cultivares utilizadas em diferentes épocas, possui o inconveniente de exigir a condução de um ensaio apenas com essa finalidade, em várias localidades e, ainda, necessita que as cultivares mais antigas estejam armazenadas, sem alterações genéticas.

Foram desenvolvidos métodos para estimativa do ganho genético, em cada par de anos consecutivos (biênio), com base nos resultados das médias de produtividade das cultivares obtidas nos ensaios de competição, para saber se a troca de cultivares, de um ano para outro, resulta em ganho de produtividade (Vencovsky et al., 1988). Nesse método, a diferença entre a produtividade média dos tratamentos comuns, a cada par de anos, estima o efeito do ano, e é fundamental que haja grande número de cultivares comuns para se obter uma estimativa precisa da variação ambiental entre os anos de avaliação. O ganho genético anual é obtido pela diferença entre a produtividade média das cultivares de um ano e a do ano imediatamente anterior, excluindo-se o efeito do ano. Aplicações desse método em milho e outras culturas (Vencovsky et al., 1988; Toledo et al., 1990; Alliprandini et al., 1993; Abreu et al., 1994; Araújo, 1995; Fernandes \& Frazon, 1997; Fonseca Júnior, 1997; Amorim Neto et al., 1998; Arias \& Ramalho, 1998; Breseghello et al., 1999; Soares et al., 1999; Atroch \& Nunes, 2000; Barbosa Neto et al., 2000; Ribeiro et al., 2003) fornecem informações importantes sobre o ganho genético em anos consecutivos.

Alguns resultados são promissores, como por exemplo os de Amorim Neto et al. (1998) que, ao usar o método proposto por Vencovsky et al. (1988), estimaram um ganho genético médio anual de $0,2123 \mathrm{t} \mathrm{ha}^{-1}(5,3 \%)$ na produtividade de arroz, e um ganho total de 1,6984 t ha ${ }^{-1}$ (42,2\%) no período de 1981/1992. Esses autores relatam que, como a estimativa do ganho médio anual é superior a duas vezes o desvio padrão dos ganhos anuais, pode-se admitir que a estimativa difere de zero, sendo rejeitada, nesse caso, a hipótese de ganho médio anual nulo.

Com dados de produtividade de feijoeiro (Phaseolus vulgaris) no Estado do Paraná, Fonseca Júnior (1997) estimou o ganho genético, entre 1977 e 1995, por seis métodos diferentes, dois existentes, dois adaptados e dois alternativos. Com relação ao método original (Vencovsky et al., 1988), empregou-se o teste t para verificar se o ganho genético médio anual foi significativo. Nesse teste, a estimativa da variância da média da estimativa do ganho foi obtida como a variância entre as estimativas dos ganhos anuais, dividida pelo número de anos, ignorando-se, portanto, os erros experimentais dos ensaios de cada ano. Outros métodos foram usados por Fonseca Júnior (1997) para estimar o ganho genético médio em uma seqüência de anos, mediante a utilização de quadrados mínimos ponderados pelas variâncias dos erros experimentais, porém a significância do ganho genético estimado num dado biênio não foi testada.

Os objetivos deste trabalho foram desenvolver e avaliar um teste de hipótese, para verificar se as substituições anuais de cultivares, em ensaios de competição de cultivares indicadas de milho, resultam em ganhos genéticos de produtividade de grãos.

\section{Material e Métodos}

Ensaios de competição de cultivares de milho de ciclo superprecoce, precoce e normal foram conduzidos na área experimental do Departamento de Fitotecnia da Universidade Federal de Santa Maria, nos anos agrícolas 1998/1999, 1999/2000, 2000/2001 e 2001/2002. A área está incluída na região fisiográfica da Depressão Central do Estado do Rio Grande do Sul, com coordenadas geográficas de $29^{\circ} 45^{\prime} \mathrm{S}$ e $53^{\circ} 42^{\prime} \mathrm{W}$, em solo classificado como Brunizem hidromórfico pertencente à Unidade de Mapeamento Santa Maria (Embrapa, 1999).

O delineamento adotado foi o de blocos ao acaso, com três repetições; a unidade experimental foi composta por duas fileiras de $5 \mathrm{~m}$ espaçadas em $0,8 \mathrm{~m}$. As sementes das cultivares, de cada ano agrícola, foram fornecidas pela Fundação Estadual de Pesquisa Agropecuária (Fepagro, RS). De cada um dos 12 ensaios (três ciclos x quatro anos), foram usados a média da produtividade de grãos a $13 \%$ de umidade de cada cultivar, o quadrado médio do erro e o grau de liberdade do erro.

Para estimar o ganho genético de um dado biênio e desenvolver o teste de hipótese, foram consideradas as estatísticas da Tabela 1. A estimativa do ganho genético ( $\hat{G} g)$, decorrente da substituição de cultivares do ano 1 para o ano 2 e, assim, para qualquer seqüência de dois anos, foi obtida pela expressão $\hat{\mathrm{G} g}=\left(\overline{\mathrm{Y}}_{2}-\overline{\mathrm{Y}}_{1}\right)-\left(\overline{\mathrm{Y}}_{\mathrm{c}_{2}}-\overline{\mathrm{Y}} \mathrm{c}_{1}\right)$ (Vencovsky et al., 1988; Fonseca Júnior, 1997).

A proposta de teste de hipótese deste trabalho admite que, sobre a hipótese de o ganho genético de um dado biênio ser nulo (H0: Gg = 0), a estatística "estimativa do 
ganho genético dividido pelo desvio padrão da estimativa do ganho genético" tem distribuição t de Student. Considerando-se que os erros referentes às estimativas de $\bar{Y}_{1}$ com $\bar{Y}_{c_{1}}$ e de $\bar{Y}_{2}$ com $\bar{Y} c_{2}$ são correlacionados, pois $\overline{\mathrm{Y}} \mathrm{c}_{1}$ está contida em $\overline{\mathrm{Y}}_{1}$, e $\overline{\mathrm{Y}} \mathrm{c}_{2}$ está contida em $\overline{\mathrm{Y}}_{2}$, a estimativa do ganho genético deve ser reorganizada. Assim, foram usadas as estatísticas em que:

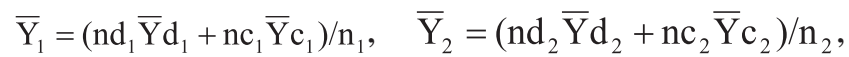
$\mathrm{n}_{1}=\mathrm{nc}_{1}+\mathrm{nd}_{1} \mathrm{e} \mathrm{n}_{2}=\mathrm{nc}_{2}+\mathrm{nd}_{2}$ para substituir na expressão do ganho genético. Tem-se, então, a estimativa do ganho genético com termos mutuamente exclusivos (independentes):

$\hat{\mathrm{G}} \mathrm{g}=\frac{\mathrm{nd}_{2}}{\mathrm{n}_{2}} \overline{\mathrm{Y}} \mathrm{d}_{2}+\frac{\mathrm{nc}_{2}}{\mathrm{n}_{2}} \overline{\mathrm{Y}} \mathrm{c}_{2}-\frac{\mathrm{nd}_{1}}{\mathrm{n}_{1}} \overline{\mathrm{Y}} \mathrm{d}_{1}-\frac{\mathrm{nc}_{1}}{\mathrm{n}_{1}} \overline{\mathrm{Y}} \mathrm{c}_{1}-\overline{\mathrm{Y}} \mathrm{c}_{2}+\overline{\mathrm{Y}} \mathrm{c}_{1}$

$=\left(1-\frac{n c_{1}}{n_{1}}\right) \bar{Y} c_{1}-\left(1-\frac{n c_{2}}{n_{2}}\right) \bar{Y} c_{2}+\frac{n d_{2}}{n_{2}} \bar{Y} d_{2}-\frac{n d_{1}}{n_{1}} \bar{Y} d_{1}$

$=\frac{\mathrm{n}_{1}-\mathrm{nc}_{1}}{\mathrm{n}_{1}} \overline{\mathrm{Y}} \mathrm{c}_{1}-\frac{\mathrm{n}_{2}-\mathrm{nc}_{2}}{\mathrm{n}_{2}} \overline{\mathrm{Y}} \mathrm{c}_{2}+\frac{\mathrm{n}_{2}-\mathrm{nc}_{2}}{\mathrm{n}_{2}} \overline{\mathrm{Y}} \mathrm{d}_{2}-\frac{\mathrm{n}_{1}-\mathrm{nc}_{1}}{\mathrm{n}_{1}} \overline{\mathrm{Y}} \mathrm{d}_{1}$

Aplicando-se o método da esperança matemática e as propriedades da variância, obtém-se a estimativa da variância da estimativa do ganho genético, ou seja,

$\hat{\mathrm{V}}(\hat{\mathrm{G} g} \mathrm{~g})=\left(\frac{\mathrm{n}_{1}-\mathrm{nc}_{1}}{\mathrm{n}_{1}}\right)^{2} \hat{\mathrm{V}}\left(\overline{\mathrm{Y}} \mathrm{c}_{1}\right)+\left(\frac{\mathrm{n}_{2}-\mathrm{nc}_{2}}{\mathrm{n}_{2}}\right)^{2} \hat{\mathrm{V}}\left(\overline{\mathrm{Y}} \mathrm{c}_{2}\right)+$

$\left(\frac{\mathrm{n}_{2}-\mathrm{nc}_{2}}{\mathrm{n}_{2}}\right)^{2} \hat{\mathrm{V}}\left(\overline{\mathrm{Y}} \mathrm{d}_{2}\right)+\left(\frac{\mathrm{n}_{1}-\mathrm{nc}_{1}}{\mathrm{n}_{1}}\right)^{2} \hat{\mathrm{V}}\left(\overline{\mathrm{Y}} \mathrm{d}_{1}\right)$

$=\left(\frac{\mathrm{n}_{1}-\mathrm{nc}_{1}}{\mathrm{n}_{1}}\right)^{2} \frac{\mathrm{QME}_{1}}{\mathrm{~J}_{1} \mathrm{nc}_{1}}+\left(\frac{\mathrm{n}_{2}-\mathrm{nc}_{2}}{\mathrm{n}_{2}}\right)^{2} \frac{\mathrm{QME}_{2}}{\mathrm{~J}_{2} \mathrm{nc}_{2}}+\left(\frac{\mathrm{n}_{2}-\mathrm{nc}_{2}}{\mathrm{n}_{2}}\right)^{2} \frac{\mathrm{QME}_{2}}{\mathrm{~J}_{2} \mathrm{nd}_{2}}+$

$\left(\frac{\mathrm{n}_{1}-\mathrm{nc}_{1}}{\mathrm{n}_{1}}\right)^{2} \frac{\mathrm{QME}_{1}}{\mathrm{~J}_{1} \mathrm{nd}_{1}}$

$=\frac{\mathrm{QME}_{1}}{\mathrm{~J}_{1}}\left(\frac{\mathrm{n}_{1}-\mathrm{nc}_{1}}{\mathrm{n}_{1}}\right)^{2}\left(\frac{1}{\mathrm{nc}_{1}}+\frac{1}{\mathrm{nd}_{1}}\right)+\frac{\mathrm{QME}_{2}}{\mathrm{~J}_{2}}\left(\frac{\mathrm{n}_{2}-\mathrm{nc}_{2}}{\mathrm{n}_{2}}\right)^{2}\left(\frac{1}{\mathrm{nc}_{2}}+\frac{1}{\mathrm{nd}_{2}}\right)$

$=\frac{\mathrm{QME}_{1}}{\mathrm{~J}_{1}}\left(\frac{1}{\mathrm{nc}_{1}}-\frac{1}{\mathrm{n}_{1}}\right)+\frac{\mathrm{QME}_{2}}{\mathrm{~J}_{2}}\left(\frac{1}{\mathrm{nc}_{2}}-\frac{1}{\mathrm{n}_{2}}\right)$.

Tabela 1. Estatísticas usadas para a definição do teste de hipótese do ganho genético num dado biênio.

\begin{tabular}{lcc}
\hline Descrição & \multicolumn{2}{c}{ Estatística } \\
\cline { 2 - 3 } & $\mathrm{Ano1}$ & $\mathrm{Ano} 2$ \\
\hline Número total de cultivares no ensaio & $\mathrm{n}_{1}$ & $\mathrm{n}_{2}$ \\
Número de cultivares comuns nos ensaios do ano 1 e 2 & $\mathrm{nc}_{1}$ & $\mathrm{nc}_{2}$ \\
Número de cultivares diferentes nos ensaios do ano 1 e 2 & $\mathrm{nd}_{1}$ & $\mathrm{nd}_{2}$ \\
Média de todas as cultivares no ensaio & $\overline{\mathrm{Y}}_{1}$ & $\overline{\mathrm{Y}}_{2}$ \\
Média das cultivares comuns nos ensaios dos anos 1 e 2 & $\overline{\mathrm{Y}} \mathrm{c}_{1}$ & $\overline{\mathrm{Y}} \mathrm{c}_{2}$ \\
Média das cultivares diferentes nos ensaios dos anos 1 e 2 & $\overline{\mathrm{Y}} \mathrm{d}_{1}$ & $\overline{\mathrm{Y}} \mathrm{d}_{2}$ \\
Quadrado médio do erro & $\mathrm{QME}_{1}$ & $\mathrm{QME}_{2}$ \\
Graus de liberdade do erro & $\mathrm{GLE}_{1}$ & $\mathrm{GLE}_{2}$ \\
Número de repetições & $\mathrm{J}_{1}$ & $\mathrm{~J}_{2}$ \\
\hline
\end{tabular}

Consideraram-se atendidos os pressupostos de normalidade, independência e homogeneidade das variâncias do erro, sob a hipótese $\mathrm{H} 0, \mathrm{Gg}=0$, a estatística $\mathrm{t}_{\mathrm{c}}=\frac{\hat{\mathrm{G} g}}{\sqrt{\hat{\mathrm{V}}(\hat{\mathrm{G} g})}} \stackrel{\mathrm{H}^{\mathrm{H}}}{\cap} \mathrm{t}_{(\mathrm{GL})}$ com GL $=\mathrm{GLE}_{1}+\mathrm{GLE}_{2}$ graus de liberdade.

A estimativa do ganho genético médio (Ĝgm), para uma sequência de $\mathrm{k}=1,2, \ldots, \mathrm{K}$ biênios, foi obtida pela expressão $\hat{\mathrm{G} g m}=\sum_{\mathrm{k}=1}^{\mathrm{K}} \hat{\mathrm{G} g} \mathrm{k} / \mathrm{K}$, cuja estimativa da variância é obtida por $\hat{\mathrm{V}}(\hat{\mathrm{G} g m})=\sum_{\mathrm{k}=1}^{\mathrm{K}} \hat{\mathrm{V}}\left(\hat{\mathrm{G}} \mathrm{g}_{\mathrm{k}}\right) / \mathrm{K}^{2}$. Sob a hipótese H0, Ggm $=0$, a estatística $t_{c}=\frac{\hat{\text { Ggm }}}{\sqrt{\hat{V}(\hat{\mathrm{G} g m})}} \stackrel{\text { H0 }}{\sim \mathrm{t}_{(\mathrm{GL})}}$ com GL = soma dos graus de liberdade dos erros de todos os experimentos.

A taxa de cultivares mantidas (TM), na seqüência de dois anos, foi estimada pela razão $\mathrm{TM}=\mathrm{nc} /\left(\mathrm{n}_{1}+\mathrm{n}_{2^{-}}\right.$ nc)x100, em que nc é o número de cultivares comuns nos ensaios dos anos 1 e 2; $\mathrm{n}_{1}$ e $\mathrm{n}_{2}$ são os números de cultivares nos ensaios dos anos 1 e 2. A taxa de substituição de cultivares (TS) foi obtida pela expressão: TS = (CN/C)x100, em que CN é o número de cultivares novas e $\mathrm{C}$ é o número total de cultivares do ano 2.

\section{Resultados e Discussão}

A amplitude das médias de rendimento de grãos entre as cultivares superprecoces $\left(6,402 \mathrm{t} \mathrm{ha}^{-1}\right)$, precoces $\left(5,356 \mathrm{t} \mathrm{ha}^{-1}\right)$ e normais $\left(5,878 \mathrm{t} \mathrm{ha}^{-1}\right)$, nos quatro anos (Tabelas 2, 3 e 4), é atribuída à substituição de cultivares e à variação ambiental entre anos e, em parte, à interação entre cultivares e anos. A diferença entre as médias das cultivares comuns, que estima o efeito ambiental para um biênio (Tabela 5), não é proporcional em todas as cultivares comuns no biênio, causando interação, o que pode mascarar a estimativa do ganho anual decorrente da substituição de cultivares. Como exemplo, no terceiro biênio do ciclo precoce (Tabela 5), a média das cultivares comuns diminuiu de $8,671 \mathrm{t} \mathrm{ha}^{-1}$ no ano 2000/2001, para 8,389 t ha-1 no ano 2001/2002 (-3\%); as cultivares AG6016 e AG6018 apresentaram redução (-8\% e -19\%, respectivamente) e a cultivar DAS8330 apresentou acréscimo (+20\%) de produtividade (Tabela 2).

A baixa taxa de manutenção de cultivares observada nos diferentes biênios, ciclos e no conjunto (Tabela 5), 
possibilitou estimar o ganho genético anual decorrente da substituição das cultivares. A menor média de taxa de substituição de cultivares entre anos foi de $53 \%$ (precoce) e a maior $65 \%$ (normal). Essas taxas estão próximas às encontradas por Arias \& Ramalho (1998) no Mato Grosso do Sul que, numa seqüência de oito anos, obtiveram taxa de substituição de 55,7\%. Essas taxas refletem o dinamismo dos programas de melhoramento de milho.

A substituição de cultivares resultou em perdas e em ganhos genéticos estimados nos diferentes ciclos e biênios. A maior perda genética ocorreu com a substituição de cultivares de ciclo normal, no biênio 2

Tabela 2. Médias do rendimento de grãos ( $\left.\mathrm{t} \mathrm{ha}^{-1}\right)$ das cultivares de ciclo superprecoce, em quatro anos agrícolas.

\begin{tabular}{|c|c|c|c|c|}
\hline Cultivar & $1998 / 1999$ & $1999 / 2000$ & $2000 / 2001$ & $2001 / 2002$ \\
\hline AG3010 & 5,040 & - & - & - \\
\hline AG6014 & 4,830 & - & - & - \\
\hline AG6016 & 5,070 & 8,827 & 9,308 & 8,596 \\
\hline AG6018 & 5,080 & 7,967 & 9,110 & 7,426 \\
\hline AG9010 & - & - & - & 7,175 \\
\hline AG9012 & 4,500 & - & - & - \\
\hline AG9014 & 5,030 & - & - & - \\
\hline AG9020 & 4,750 & - & - & - \\
\hline AGN2012 & - & 7,293 & 6,100 & - \\
\hline AGN3050 & - & 8,187 & 7,622 & - \\
\hline AGN3060 & - & 6,020 & - & - \\
\hline AGN3100 & - & 6,740 & - & - \\
\hline AGN3150 & - & 5,580 & 4,162 & - \\
\hline AGN3180 & - & 7,614 & - & - \\
\hline AGN3511 & - & - & 6,651 & - \\
\hline AGROM2012 & 5,270 & - & - & - \\
\hline AGROM3050 & 3,820 & - & - & - \\
\hline AGROM3060 & 4,720 & - & - & - \\
\hline AGROM3100 & 4,070 & - & - & - \\
\hline AGROM 3150 & 3,230 & - & - & - \\
\hline AGROM3180 & 3,770 & - & - & - \\
\hline AS3601 & - & 7,004 & 5,589 & - \\
\hline C801 & - & 7,280 & - & - \\
\hline C806 & 4,780 & 8,566 & 7,351 & - \\
\hline C855 & 4,180 & 7,400 & - & - \\
\hline С909 & 5,440 & 7,219 & 7,529 & - \\
\hline C929 & - & - & 6,751 & - \\
\hline CD3211 & - & 6,950 & 5,069 & - \\
\hline $\mathrm{CO} 32$ & 5,130 & - & - & 7,415 \\
\hline DAS766 & - & - & - & 8,937 \\
\hline DAS 8330 & - & - & 7,596 & 9,144 \\
\hline DAS 9560 & - & - & - & 8,653 \\
\hline DENSUS & 3,190 & - & - & - \\
\hline DINA657 & 4,180 & - & 4,810 & - \\
\hline DINA769 & 4,630 & - & - & - \\
\hline DINACO 9560 & - & - & 8,152 & - \\
\hline DKB440 & - & - & - & 7,547 \\
\hline DKB909 & - & - & - & 6,935 \\
\hline DOMINIUM & 3,730 & - & - & - \\
\hline FLASH & - & - & 9,019 & - \\
\hline P32R21 & - & 9,592 & 9,546 & - \\
\hline SAVE394 & - & - & - & 6,218 \\
\hline SHS4050 & - & 7,427 & - & 7,406 \\
\hline SHS5050 & - & 7,925 & - & 7,913 \\
\hline SHS5070 & - & 7,639 & - & 6,491 \\
\hline XL205 & 3,710 & 8,573 & - & - \\
\hline XL220 & - & 8,801 & - & - \\
\hline XL321 & 5,210 & - & - & - \\
\hline XL330 & 4,980 & - & - & - \\
\hline Z8392 & 4,040 & - & - & - \\
\hline
\end{tabular}

$\left(-0,947 t \mathrm{ha}^{-1}\right)$, o que indica que a taxa de substituição de $67 \%$ foi significativamente ineficiente. O maior ganho

Tabela 3. Médias do rendimento de grãos ( $\left(\mathrm{h} \mathrm{ha}^{-1}\right)$ das cultivares de ciclo precoce, em quatro anos agrícolas.

\begin{tabular}{|c|c|c|c|c|}
\hline Cultivar & $1998 / 1999$ & $1999 / 2000$ & $2000 / 2001$ & $2001 / 2002$ \\
\hline P30F33 & - & - & 7,570 & - \\
\hline 8410 & - & - & 6,288 & - \\
\hline 8420 & - & - & 8,368 & - \\
\hline 8447 & - & - & $\begin{array}{l}6,098 \\
6,098\end{array}$ & 5,574 \\
\hline $\mathrm{AG} 122$ & 5,040 & - & 7,436 & 5,865 \\
\hline AG303 & 4,620 & - & 7,220 & 5,817 \\
\hline AG5011 & 5,550 & - & 7,190 & 6,427 \\
\hline AG5014 & 5,310 & - & - & - \\
\hline AG519 & 3,950 & - & - & - \\
\hline AG8014 & 5,240 & - & - & - \\
\hline AG9090 & - & - & - & 7,291 \\
\hline AGN2003 & - & 3,908 & - & - \\
\hline AGN2012 & - & - & - & 5,619 \\
\hline AGN2511 & - & - & 7,775 & - \\
\hline AGN25A11 & - & - & - & 5,947 \\
\hline AGN3050 & - & - & - & 6,510 \\
\hline AGN3100 & - & - & 7,009 & 5,099 \\
\hline AGN3150 & - & - & - & 6,038 \\
\hline AGN3180 & - & - & 5,733 & 5,424 \\
\hline AGN35A11 & - & - & - & 6,634 \\
\hline AGROM2003 & 4,150 & - & - & - \\
\hline AGROM2010 & 4,010 & - & - & - \\
\hline AS1533 & - & - & - & 6,446 \\
\hline AS32 & 4,770 & 6,989 & 6,441 & - \\
\hline AS3466 & 4,630 & 5,400 & - & - \\
\hline AS 3477 & - & 6,295 & 7,936 & 6,188 \\
\hline AS523 & 5,130 & 6,694 & 7,848 & 6,457 \\
\hline ATTACK & - & - & - & 6,492 \\
\hline AVANT & 6,140 & 5,786 & - & - \\
\hline BRS2160 & - & - & 7,278 & - \\
\hline BRS3060 & - & - & - & 4,663 \\
\hline $\mathrm{C} 435$ & - & 5,313 & 6,286 & - \\
\hline $\mathrm{C} 444$ & - & 5,935 & - & - \\
\hline C701 & 4,430 & - & 6,087 & - \\
\hline CD301 & - & - & 6,348 & - \\
\hline CD3121 & - & - & 6,677 & 6,466 \\
\hline CEP304 & 3,980 & - & - & - \\
\hline $\mathrm{CO} 32$ & - & 6,595 & - & - \\
\hline D1000 & 5,020 & 7,181 & - & - \\
\hline DAS657 & - & - & - & 6,988 \\
\hline DAS 8460 & - & - & - & 6,184 \\
\hline DAS 8480 & - & - & - & 6,944 \\
\hline DINA170 & 4,480 & - & - & - \\
\hline DINA566 & 6,100 & - & - & - \\
\hline DINA766 & 6,340 & - & 7,391 & - \\
\hline DKB280 & - & - & 7,256 & 7,302 \\
\hline DKB435 & - & - & - & 6,010 \\
\hline DKB701 & - & - & - & 6,287 \\
\hline DKB747 & - & - & - & 6,669 \\
\hline G800 & 5,600 & 6,832 & - & - \\
\hline $\mathrm{G} 8447 \mathrm{G}$ & - & 5,248 & - & - \\
\hline PREMIUM & 6,060 & 7,377 & 9,264 & 6,128 \\
\hline SG150 & 5,610 & - & - & 6,401 \\
\hline SHS4040 & - & - & 5,792 & 5,996 \\
\hline SHS4050 & - & - & 7,361 & - \\
\hline SHS5050 & - & - & 7,628 & - \\
\hline SHS5060 & - & 5,874 & 6,725 & 5,447 \\
\hline SHS5070 & - & - & 7,364 & - \\
\hline STAR & 5,350 & - & - & - \\
\hline TORK & 6,310 & 6,991 & 9,068 & 7,714 \\
\hline XL212 & 5,930 & 6,381 & - & - \\
\hline XL214 & 6,030 & 7,050 & 8,739 & - \\
\hline XL215 & 5,010 & 6,512 & 7,495 & - \\
\hline XL340 & 5,890 & 4,679 & - & - \\
\hline XL344 & - & 4,814 & 7,614 & - \\
\hline XL345 & 5,270 & 5,656 & - & - \\
\hline Z8440 & 4,290 & - & - & - \\
\hline Z8474 & 5,030 & 7,453 & - & - \\
\hline Z8486 & - & 5,058 & - & - \\
\hline
\end{tabular}


genético foi de $0,815 \mathrm{t} \mathrm{ha}^{-1}$, obtido nas cultivares superprecoces do biênio 3. Nesse caso, considerandose a não significância, mostra-se a pouca eficiência da taxa de substituição de $85 \%$. No entanto, a baixa taxa de manutenção (11\%) pode não refletir a verdadeira variação ambiental, mascarada pela interação entre anos e cultivares comuns. Na média dos três biênios, houve um ganho genético não significativo de $0,222 \mathrm{t} \mathrm{ha}^{-1}$, para cultivares classificadas como de ciclo superprecoce, e uma perda genética não significativa de 0,070 e $0,067 \mathrm{t} \mathrm{ha}^{-1}$, para cultivares de ciclo precoce e normal, respectivamente.

Os resultados da análise dos pressupostos de normalidade e independência dos erros e da homogeneidade das variâncias, nos ensaios do mesmo ciclo, levam a deduzir que não há impedimentos para a aplicação dos testes de hipótese, apresentados na Tabela 5 . O teste de hipótese bilateral, que considerou a hipótese nula (H0: $\mathrm{Gg}=0)$ contra a alternativa $\left(\mathrm{H}_{1}: \mathrm{Gg} \neq 0\right.$ ), revelou que, a $5 \%$ de probabilidade, a substituição de cultivares não foi eficiente. Além disso, as cultivares de ciclo normal incluídas no biênio 2 foram ineficientes, podendo-se afirmar, a 5\% de probabilidade, que ocasionaram perdas genéticas. Nos demais sete casos, os ganhos ou perdas genéticas foram aleatórios, e não resultantes da substituição de cultivares (Tabela 5).

A análise conjunta de todas as cultivares, independentemente do ciclo, mostrou um ganho genético médio anual de $0,175 \mathrm{t} \mathrm{ha}^{-1}$ durante o período, equivalente a

Tabela 4. Médias do rendimento de grãos ( $\left.\mathrm{t} \mathrm{ha} \mathrm{a}^{-1}\right)$ das cultivares de ciclo normal, em quatro anos agrícolas.

\begin{tabular}{lcccc}
\hline Cultivar & $1998 / 1999$ & $1999 / 2000$ & $2000 / 2001$ & $2001 / 2002$ \\
\hline 3232 & 4,710 & - & - & - \\
AG1043 & 3,920 & - & - & - \\
AG1051 & 4,430 & 7,666 & 5,710 & 7,289 \\
AG1061 & 5,490 & - & - & - \\
AG4051 & 4,960 & - & - & - \\
AGN12A25 & - & - & - & 7,325 \\
AGROM1030 & 4,950 & - & - & - \\
B670 & - & 8,978 & - & - \\
C333B & - & 6,649 & - & - \\
CDX98T04 & - & - & 4,460 & - \\
FT9043 & 3,100 & - & - & - \\
GRAUNA133 & - & - & - & 5,978 \\
MTC828 & - & - & 5,020 & - \\
OC705 & 3,870 & 5,216 & 4,680 & 7,113 \\
P3232 & - & 7,446 & 6,110 & 7,082 \\
R-3021 & - & - & 4,730 & - \\
R-8550 & - & - & 5,450 & - \\
R-85E03 & - & - & 5,300 & - \\
R-AG8080 & - & - & - & 6,178 \\
R-BRS2160 & - & - & - & 5,452 \\
R-CD303 & - & - & 5,930 & 7,402 \\
R-DAS8550 & - & - & - & 7,823 \\
XL370 & - & 8,417 & - & - \\
XL660 & - & 8,054 & - & - \\
XL678 & 4,350 & - & - & - \\
\hline
\end{tabular}

$3,6 \%$ em relação à média geral de produtividade no primeiro ano $\left(4,804 \mathrm{t} \mathrm{ha}^{-1}\right)$. Nessa análise houve um ganho genético no primeiro e terceiro biênios, significativo a $5 \%$ de probabilidade, e perda de $0,067 \mathrm{t} \mathrm{ha}{ }^{-1}$ não significativa no segundo ano. A produtividade média de milho, no Rio Grande do Sul, é de aproximadamente $3 \mathrm{t} \mathrm{ha} \mathrm{H}^{-1}$, o que leva a inferir que, de maneira geral, os programas de melhoramento de cultivares de milho vêm substituindo as cultivares de forma eficiente e que, no caso desta

Tabela 5. Anos agrícolas, biênios, número de cultivares (n), número de cultivares comuns (nc), média de todas as cultivares $(\overline{\mathrm{Y}})$, média das cultivares comuns $(\overline{\mathrm{Y}} \mathrm{c})$, quadrado médio do erro (QME), graus de liberdade do erro (GLE), estimativa do ganho genético ( $\hat{\mathrm{G} g}$ ) para produtividade de grãos de milho ( $\mathrm{t} \mathrm{ha}^{-1}$ ), taxa de cultivares mantidas (TM), taxa de cultivares substituídas (TS) e estimativa da variância de $\hat{\mathrm{G} g}[\hat{\mathrm{V}}(\hat{\mathrm{G} g} \mathrm{~g})]$, nos diferentes ciclos e no conjunto.

\begin{tabular}{|c|c|c|c|c|c|c|c|}
\hline \multirow[t]{2}{*}{ Estatísticas } & \multicolumn{2}{|c|}{ Biênio 1} & \multicolumn{2}{|c|}{ Biênio 2} & \multicolumn{2}{|c|}{ Biênio 3} & \multirow[t]{2}{*}{ Média } \\
\hline & $\begin{array}{l}1998 / 1999 \\
\end{array}$ & $1999 / 2000$ & 1999/2000 & $2000 / 2001$ & $2000 / 200$ & $2001 / 2002$ & \\
\hline & \multicolumn{7}{|c|}{ Ciclo superprecoce } \\
\hline $\mathrm{n}$ & 25 & 20 & 20 & 16 & 16 & 13 & - \\
\hline $\mathrm{nc}$ & 6 & 6 & 10 & 10 & 3 & 3 & - \\
\hline$\overline{\mathrm{Y}}$ & 4,495 & 7,630 & 7,630 & 7,148 & 7,148 & 7,681 & - \\
\hline$\overline{\mathrm{Y}} \mathrm{c}$ & 4,710 & 8,092 & 7,719 & 7,139 & 8,671 & 8,389 & - \\
\hline QME & 1,010 & 1,442 & 1,442 & 1,713 & 1,713 & 0,922 & - \\
\hline GLE & 48 & 38 & 38 & 30 & 30 & 24 & - \\
\hline$\hat{\mathrm{Gg}}$ & \multicolumn{2}{|c|}{$-0,247^{\mathrm{ns}}$} & & $0,098^{\text {ns }}$ & & $0,815^{\mathrm{ns}}$ & $0,222^{\mathrm{ns}}$ \\
\hline TM $(\%)$ & \multicolumn{2}{|c|}{15} & & 38 & & 11 & 21 \\
\hline TS (\%) & \multicolumn{2}{|c|}{$\begin{array}{c}70 \\
0.0987\end{array}$} & & 38 & & 69 & 59 \\
\hline \multirow[t]{2}{*}{$\hat{\mathrm{V}}(\hat{\mathrm{G} g} \mathrm{~g}$} & \multicolumn{2}{|c|}{0,0987} & & 0,0454 & & 0,2334 & 0,0419 \\
\hline & \multicolumn{7}{|c|}{ Ciclo precoce } \\
\hline $\mathrm{n}$ & 30 & 23 & 23 & 30 & 30 & 30 & - \\
\hline nc & 14 & 14 & 10 & 10 & 14 & 14 & - \\
\hline$\overline{\mathrm{Y}}$ & 5,176 & 6,088 & 6,088 & 7,243 & 7,243 & 6,234 & - \\
\hline$\overline{\mathrm{Y}} \mathrm{c}$ & 5,487 & 6,499 & 6,391 & 7,742 & 7,232 & 6,136 & - \\
\hline QME & 0,408 & 0,798 & 0,798 & 0,708 & 0,708 & 0,830 & - \\
\hline GLE & 58 & 44 & 44 & 58 & 58 & 58 & - \\
\hline$\hat{\mathrm{G} g}$ & \multicolumn{2}{|c|}{$-0,100^{\mathrm{ns}}$} & \multicolumn{2}{|c|}{$-0,196^{\mathrm{ns}}$} & \multicolumn{2}{|c|}{$0,087^{\mathrm{ns}}$} & $-0,070^{\mathrm{n}}$ \\
\hline $\mathrm{TM}(\%)$ & \multicolumn{2}{|c|}{36} & \multirow{2}{*}{\multicolumn{2}{|c|}{23}} & \multirow{2}{*}{\multicolumn{2}{|c|}{30}} & 30 \\
\hline $\mathrm{TS}(\%)$ & \multirow{2}{*}{\multicolumn{2}{|c|}{$\begin{array}{c}39 \\
0,0126\end{array}$}} & & & & & 53 \\
\hline \multirow[t]{2}{*}{$\hat{\mathrm{V}}(\hat{\mathrm{G} g})$} & & & \multicolumn{2}{|c|}{0,0308} & \multicolumn{2}{|c|}{0,0195} & 0,0069 \\
\hline & & & & lo normal & & & \\
\hline $\mathrm{n}$ & 9 & 7 & 7 & 9 & 9 & 9 & - \\
\hline nc & 2 & 2 & 3 & 3 & 4 & 4 & - \\
\hline $\bar{Y}$ & 4,420 & 7,489 & 7,489 & 5,266 & 5,266 & 6,849 & - \\
\hline$\overline{\mathrm{Y}} \mathrm{c}$ & 4,150 & 6,441 & 6,776 & 5,500 & 5,608 & 7,222 & - \\
\hline QME & 0,687 & 0,601 & 0,601 & 0,813 & 0,813 & 0,459 & - \\
\hline GLE & 16 & 12 & 12 & 16 & 16 & 16 & - \\
\hline$\hat{\mathrm{G} g}$ & & $78^{\mathrm{ns}}$ & -0 & & & & $-0,067^{\mathrm{n}}$ \\
\hline $\mathrm{TM}(\%)$ & & & & & & & 22 \\
\hline $\mathrm{TS}(\%)$ & & & & & & & 65 \\
\hline$\hat{\mathrm{V}}(\hat{\mathrm{G} g})$ & & & & & & & 0,0353 \\
\hline & & & & Onjunto & & & \\
\hline $\mathrm{n}$ & 64 & 50 & 50 & 55 & 55 & 52 & - \\
\hline nc & 23 & 23 & 28 & 28 & 26 & 26 & - \\
\hline $\bar{Y}$ & 4,804 & 6,901 & 6,901 & 6,892 & 6,892 & 6,702 & - \\
\hline$\overline{\mathrm{Y}} \mathrm{c}$ & 5,153 & 6,913 & 7,099 & 7,157 & 7,013 & 6,569 & - \\
\hline QME & 0,814 & 1,086 & 1,086 & 1,104 & 1,104 & 0,804 & - \\
\hline GLE & 126 & 98 & 98 & 108 & 108 & 102 & - \\
\hline$\hat{\mathrm{Gg}}$ & 0,3 & & $-0,0$ & & & & $0,175^{*}$ \\
\hline $\mathrm{TM}(\%)$ & & & & & & & 31 \\
\hline $\mathrm{TS}(\%)$ & & & & & & & 51 \\
\hline$\hat{\mathrm{V}}(\hat{\mathrm{G} g} \mathrm{~g})$ & 0,0 & & 0,0 & & & & 0,0045 \\
\hline
\end{tabular}

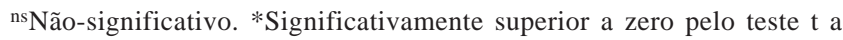
$5 \%$ de probabilidade. 
pesquisa, contribuíram para a melhoria da produtividade da cultura, numa taxa de 5,8\% ao ano.

Mudanças de cultivares de um ciclo para outro foram observadas no transcorrer dos quatro anos em que foram avaliadas, pois a taxa de cultivares mantidas (TM) conjunta foi maior que o máximo observado nos três ciclos, ou o número de cultivares comuns no conjunto é maior do que a soma do número de cultivares comuns nos três ciclos do mesmo biênio. Essas cultivares, consideradas excluídas num ciclo e incluídas no outro ciclo e ano, aparecem como comuns quando a estimativa do ganho é feita no conjunto dos três ciclos. Esse fato prejudica a estimativa real do ganho decorrente das substituições e altera as diferenças entre os ciclos e em relação ao conjunto, quando as migrações não interferem nas estimativas do ganho. Além disso, uma alta taxa de renovação de cultivares indicadas impossibilita a existência de um programa eficiente de avaliação e de indicação de cultivares.

\section{Conclusões}

1. O teste de hipótese proposto possibilita verificar se as substituições anuais de cultivares indicadas de milho são eficientes para aumentar a produtividade de grãos.

2. As substituições anuais de cultivares indicadas de milho são dinâmicas e nem sempre resultam em ganho genético para produtividade de grãos.

\section{Referências}

ABREU, A.F.B.; RAMALHO, M.A.P.; SANTOS, J.B.; MARTINS, L.A. Progresso do melhoramento genético do feijoeiro nas décadas de setenta e oitenta nas Regiões Sul e Alto Paranaíba em Minas Gerais. Pesquisa Agropecuária Brasileira, v.29, p.105-112, 1994. ALLIPRANDINI, L.F.; TOLEDO, J.F.F. de; FONSECA JÚNIOR, N.S.; KIIHL, R.A. de S.; ALMEIDA, L.A. de. Ganho genético em soja no Estado do Paraná, via melhoramento, no período de 1985/86 a 1989/90. Pesquisa Agropecuária Brasileira, v.28, p.489-497, 1993.

AMORIM NETO, S.; ANDRADE, W.E. de B.; COSTA, R.A. da. Aumento da produtividade de arroz irrigado no Estado do Rio de Janeiro de 1981/82 a 1991/92. Pesquisa Agropecuária Brasileira, v.33, p.369-374, 1998.

ARAÚJO, J.S. de. Ganhos genéticos obtidos em híbridos e variedades de milho representativos de três décadas de melhoramento no Brasil. 1995. 64p. Dissertação (Mestrado) Universidade Federal de Lavras, Lavras.

ARIAS, E.R.A.; RAMALHO, M.A.P. Progresso genético em milho no Estado do Mato Grosso do Sul, no período de 1986/87 a 1993/94. Pesquisa Agropecuária Brasileira, v.33, p.1534-1538, 1998.
ATROCH, A.L.; NUNES, G.H. de S. Progresso genético em arroz de várzea úmida no Estado do Amapá. Pesquisa Agropecuária Brasileira, v.35, p.767-771, 2000.

BARBOSA NETO, J.F.; MATIELLO, R.R.; CARVALHO, F.I.F. de; OLIVEIRA, J.M.S.; PEGORARO, D.G.; SCHNEIDER, F.; SORDI, M.H.B.; VACARO, E. Progresso genético no melhoramento da aveia-branca no Sul do Brasil. Pesquisa Agropecuária Brasileira, v.35, p.1605-1612, 2000.

BRESEGHELLO, F.; RANGEL, P.H.N.; MORAIS, O.P. de. Ganho de produtividade pelo melhoramento genético do arroz irrigado no Nordeste do Brasil. Pesquisa Agropecuária Brasileira, v.34, p.399407, 1999.

EMBRAPA. Centro Nacional de Pesquisa de Solos. Sistema Brasileiro de classificação de solos. Brasília: Embrapa-SPI, 1999. 412p.

FERNANDES, J.S.C. Estabilidade ambiental de cultivares de milho na Região Centro Sul do Brasil. 1988. 94p. Dissertação (Mestrado) - Escola Superior de Agricultura “Luiz de Queiroz”, Piracicaba.

FERNANDES, J.S.C.; FRAZON, J.F. Thirty years of genetic progress in maize (Zea mays L.) in a tropical environment. Maydica, v.42, p.21-27, 1997.

FONSECA JÚNIOR, N.S. Progresso genético na cultura do feijão no Estado do Paraná para o período de 1977 a 1995. 1997. 168p. Tese (Doutorado) - Escola Superior de Agricultura “Luiz de Queiroz", Piracicaba.

MORAIS, O.P.; ABBUD, N.S. Subsídios para avaliação do progresso genético dos programas estaduais de melhoramento de arroz no Brasil. Goiânia, EMBRAPA-CNPAF, 1993, 26p. Apostila.

RIBEIRO, N.D.; POSSEBON, S.B.; STORCK, L. Progresso genético em caracteres agronômicos no melhoramento do feijoeiro. Ciência Rural, v.33, p.629-633, 2003.

SOARES, A.A.; SANTOS, P.G.; MORAIS, O.P. de; SOARES, P.C.; REIS, M. de S.; SOUZA, M.A. de. Progresso genético obtido pelo melhoramento do arroz de sequeiro em 21 anos de pesquisa em Minas Gerais. Pesquisa Agropecuária Brasileira, v.34, p.415424, 1999.

STORCK, L.; LÚCIO, A.D.; MARTIN, T.N. Tempo de permanência dos genótipos recomendados de milho nos ensaios de competição, no Estado do Rio Grande do Sul. In.: REUNIÃO TÉCNICAANUAL DO MILHO E SORGO, 45., 2000, Pelotas. Anais. Pelotas: Embrapa Clima Temperado, 2000, p.261-264.

TOLEDO, J.F.F. de; ALMEIDA, L.A. de; KIIHL, R.A. de S.; MENOSSO, M.G. Ganho genético em soja no Estado do Paraná, via melhoramento. Pesquisa Agropecuária Brasileira, v.25, p.89-94, 1990.

VENCOVSKY, R.; MORAIS, A.R.; GARCIA, J.C.; TEIXEIRA, N.M. Progresso genético em vinte anos de melhoramento do milho no Brasil. In: CONGRESSO NACIONAL DE MILHO E SORGO, 16., 1986, Belo Horizonte. Anais. Sete Lagoas: Embrapa-CNPMS, 1988, p.300-307. 